\title{
"Context, content, and system" supporting digital health hub (DHH)-enabled models of care (MoCs) for fragility hip fractures: perspectives of diverse multidisciplinary stakeholders in South Australia from qualitative in-depth interviews
}

\author{
Lalit Yadav $^{1,2,3} \cdot$ Tiffany K. Gill $^{3} \cdot$ Anita Taylor $^{2} \cdot$ Jennifer deYoung ${ }^{2} \cdot$ Renuka Visvanathan $^{1,3,4} \cdot$ Mellick J. Chehade $^{1,2,3}$
}

Received: 24 February 2021 / Accepted: 19 October 2021 / Published online: 6 November 2021

(c) International Osteoporosis Foundation and National Osteoporosis Foundation 2021

\begin{abstract}
Summary Combining thematic analysis and a human-computer persuasive systems framework suggests that hip fracture recovery among older people can be enhanced through person-centered digital health hub models of care focused on behavior change education and integrated care. The findings intend to guide settings involving comorbid conditions and low- and middle-income countries in developing innovative digital health solutions.

Purpose The purpose of this study was to understand stakeholders' perspectives on the development of a digital healthenabled model of care for fragility hip fractures and to map out factors that could influence the design and implementation of such a model.

Methods Qualitative in-depth interviews were conducted with stakeholders from various clinical disciplines, allied health, and computer science. A hybrid process involving thematic analysis of the raw data using inductive coding was the first step. In the second step, the tenets of a theoretical framework (health behavior change supporting systems) were deductively applied to the thematic constructs generated as part of the first step of the analysis.

Results In total, 24 in-depth interviews were conducted with stakeholders. We identified 18 thematic constructs presented under the categories of context, content, and system. Context covered patient characteristics such as frailty, digital literacy, and patient or carer participation, whereas healthcare delivery aspects included the structure and culture of existing practice and the need for innovative holistic models of care. Content outlines the active ingredients and approach in developing a digital health hub, and it highlights the importance of targeted education and behavior change. The system is a complicated matrix crossing different aspects of healthcare and offering a value proposition design through personalization across modes of content delivery. This must foster trust, ensure adequate financing, and support ownership and privacy by establishing appropriate mechanisms for embedding change.

Conclusion The findings from this study provide insights around potential factors related to patients, community support, and healthcare delivery influencing the design and next-stage implementation of a digital health hub model of care for fragility hip fractures.
\end{abstract}

Keywords Digital health $\cdot$ Models of care $\cdot$ Hip fractures $\cdot$ Qualitative method $\cdot$ Frailty $\cdot$ Multimorbidity

\section{Introduction}

Tiffany K. Gill and Mellick J. Chehade are joint senior authors

Lalit Yadav

lalit.yadav@adelaide.edu.au

Extended author information available on the last page of the article
Fragility hip fractures among older adults present a significant burden to society in terms of disability, mortality, and economic repercussions for both community and health systems [1-4]. While the rate of fragility fractures in Australia is expected to rise by at least $10 \%$ every 5 years, hip fractures are among the most devasting of these fractures, with almost $50 \%$ requiring a permanent shift to residential aged 
care within 12 months [2]. The combined health and social care costs in the first year following a hip fracture injury are enormous and unsustainable; the costs are reported to be $\$ 43,669$ on average, which is higher than equivalent estimates for ischemic stroke and acute coronary syndrome [3]. Hip fracture events are complex, manifested by disequilibrium of normal physiological processes at the individual patient level, and accompanied by inadequate management of multimorbid conditions at the health system level [1-4].

Multimorbidity is the presence of more than one chronic condition in an individual. It is influenced not only by healthrelated characteristics but also by socioeconomic, cultural, and environmental factors, as well as patient behavior [5]. It is argued that the current health system is predominantly geared toward labeling and addressing chronic disease through siloed care pathways rather than having a wider view of disease etiology and interacting factors [6]. Thus, it is vitally important to acknowledge that the management of older people with fragility hip fractures is a complex, multidimensional issue that extends beyond the acute care facility and immediate discharge care $[1,5,7]$.

Recognition of the role that patients, their family members, and other carers play as part of an ecosystem, or "networked units," is becoming critically important for improving outcomes [7-9]. Individuals have unique life circumstances influenced by differing levels of health literacy, which affects their perception of health and wellbeing and their disease outcomes [4, 9-11]. Moreover, with increasing access to information technology through computers and mobile applications, there is a growing recognition that people will become more curious to seek information related to their present health condition. Before undertaking this research, we conducted a comprehensive systematic review to evaluate the effectiveness of targeted patient communication supported through digital health interventions versus the standard provision of health information on the recovery from fragility fractures among older adults [1]. The findings from this review were divided into three categories as defined by the latest World Health Organization (WHO) guidelines on digital health interventions. A meta-analysis suggested that targeted patient communication with primary care physician support using digital health was twice as effective as the standard provision of health information, such as patient information leaflets, in preventing secondary fractures post-hospital discharge. It was further highlighted that providing health information alone does not equate to educating patients. The other two digital intervention categories also improved functional outcomes among older fragility fracture patients. However, significant challenges were involved in using sophisticated forms of interactive digital health technologies, such as personal health tracking and healthcare provider decision support. There is a need to proactively engage different stakeholders, including healthcare providers from multiple disciplines, patients, and their family members, to cocreate a digital health solution that could potentially become an integral part of local service design and delivery $[1,4,5,9]$.

This study is the first phase (formative research) of a research program aimed at informing the development of digital health-enabled models of care (MoCs) to improve patient education, functional outcomes, and quality of life and foster continuity of supported and integrated care among older people with hip fractures [4]. Our previously published protocol paper examined different theoretical frameworks around computer-human persuasive systems (Health Behavior Change Supporting Systems (HBCSS)), implementation science, and best practice guidelines on hip fracture management and the management of older people within the community $[4,12-15]$. For this research component, we utilized three constructs of HBCSS (content, context, and system) to guide the design and execution of the study. The "content" essentially refers to the evidence-based information that will be embedded into the digital health solution and the way consumers will be engaged to inform its further evolution and iteration. The "context" includes factors influencing the local setting in which we intend to develop, pilot, and later implement such a digital health solution, while the "system" encompasses the supporting infrastructure requirements, including aspects of operational mechanisms $[4,12]$.

The objectives of this study were to understand the perspectives of diverse stakeholders on the development of digital health-enabled MoCs for fragility hip fractures and to map out potential factors that could influence design and implementation within the local healthcare setting [4, 12]. Stakeholders in this study are healthcare practitioners from different clinical disciplines, allied health, health education, public health, computer science, and other fields who could influence policy and key decision-making at the local contextual level. The perspectives of patients, their family members, and residential aged carers were covered in another study [7].

\section{Methods}

\section{Study design and participants}

This study involved a wide range of stakeholders from the Central Adelaide Local Health Network (CALHN), which is responsible for promoting and improving the health of the central metropolitan Adelaide community and provides specialized care for South Australians through integrated healthcare and hospital services. Additionally, a substantial number of people from outside these geographic areas also 
access services offered by CALHN. Stakeholders in this study were healthcare practitioners from multiple disciplines at different levels, ranging from senior administration to frontline staff. Their disciplines included orthopedics, geriatrics, physiotherapy, occupational therapy, dietetics, public health, and social and computer sciences. These study participants were among the key actors who could influence the micro (local clinical practice), meso (service delivery), and macro (policy and research agenda) levels. An in-depth qualitative interview method was used to enable participants to describe their views and experiences in detail; this method has been previously described $[4,16]$. We followed the Consolidated Criteria for Reporting Qualitative Studies (COREQ), a 32-item checklist, throughout this manuscript (Supplementary 2) [17].

\section{Recruitment and setting}

A purposive sampling strategy was used, and we identified potential participants through various routes, including clinicians working in the multidisciplinary hip fracture care environment across various settings (primary, secondary, and tertiary care), researchers, and policy or decision-makers. With this sampling strategy, we selected study participants belonging to different but relevant disciplines, with different areas of subject expertise, and with varying years of experience, including some early career participants and some highly experienced participants. This approach ensured the diversity of participants in terms of their roles, professional background, and level of experience.

Participants were approached using multiple strategies, such as email, phone, or in-person invitations. Participants were provided an information sheet with a detailed description of the study and an opportunity to ask questions and written and/or verbal consent was obtained as appropriate. The principal investigator (LY) then identified a suitable time and mode for interviews with interested participants.

\section{Data collection}

An interview guide (Supplementary 1) was developed based on the contemporary literature on digital health, social and behavioral change theories, implementation science, and best practice guidelines for the management of hip fractures. This interview guide consisted of open-ended questions divided into three broad categories-content, context, and system-with embedded prompts [4]. This approach provided flexibility during the interview process to allow participants to raise issues that were personally salient to them.

\section{Data analysis}

All of the interviews were audio-recorded. LY transcribed the recordings verbatim, checked for inconsistencies, and anonymized the data. Details of individuals, places, or any other identifiable information were removed before sharing transcripts with team members. A hybrid process of inductive and deductive thematic analysis to interpret the raw data was used. This involved thematic analysis of the raw data using inductive coding as a first step and then deductively applying the tenets of HBCSS to the theme constructs generated as part of the first step of the analysis $[12,18]$. An initial coding framework was developed while the stakeholder interviews were still being conducted by identifying themes through careful reading and rereading of the first three interview transcripts. This coding framework was further tested and refined as interview transcripts aligned with emerging themes under the broad categories of content, context, and system (HBCSS), and comparisons were made within and across the interviews. The team members (LY, TG, MJC, RV) derived detailed insights and interpretations from the data considering the aims of the analysis and their knowledge of the domain and literature. Data collection ceased when emerging themes were saturated. A range of tools were adopted, using a conventional approach to coding and using modern software technology for data management. An open coding approach helped the researchers understand and search for hidden conceptual meanings, which allowed the freedom to think along different lines and develop new concepts. NVivo 12 [19] was used as a tool for data sorting and management, aiding the further analysis and interpretation of the study findings.

\section{Results}

In total, 24 in-depth interviews were conducted with stakeholders representing diverse roles and responsibilities within the CALHN in Adelaide, South Australia. The participants included 11 females and 13 males across a wide age spectrum, from their early $20 \mathrm{~s}$ to their late $60 \mathrm{~s}$. Some of them were involved in private clinical practice in addition to their role at the CALHN. Each interview lasted approximately $45 \mathrm{~min}$ to an hour on average, ranging from $30 \mathrm{~min}$ to an hour and a half. All interviews were conducted in English, and participants provided written informed consent.

The findings from this study are presented in the form of 18 distinct thematic constructs, followed by descriptions and corresponding quotes (Table 1) under three broad categories-context, content, and systems-aligning with the HBCSS framework [4, 12]. The results presented in this 


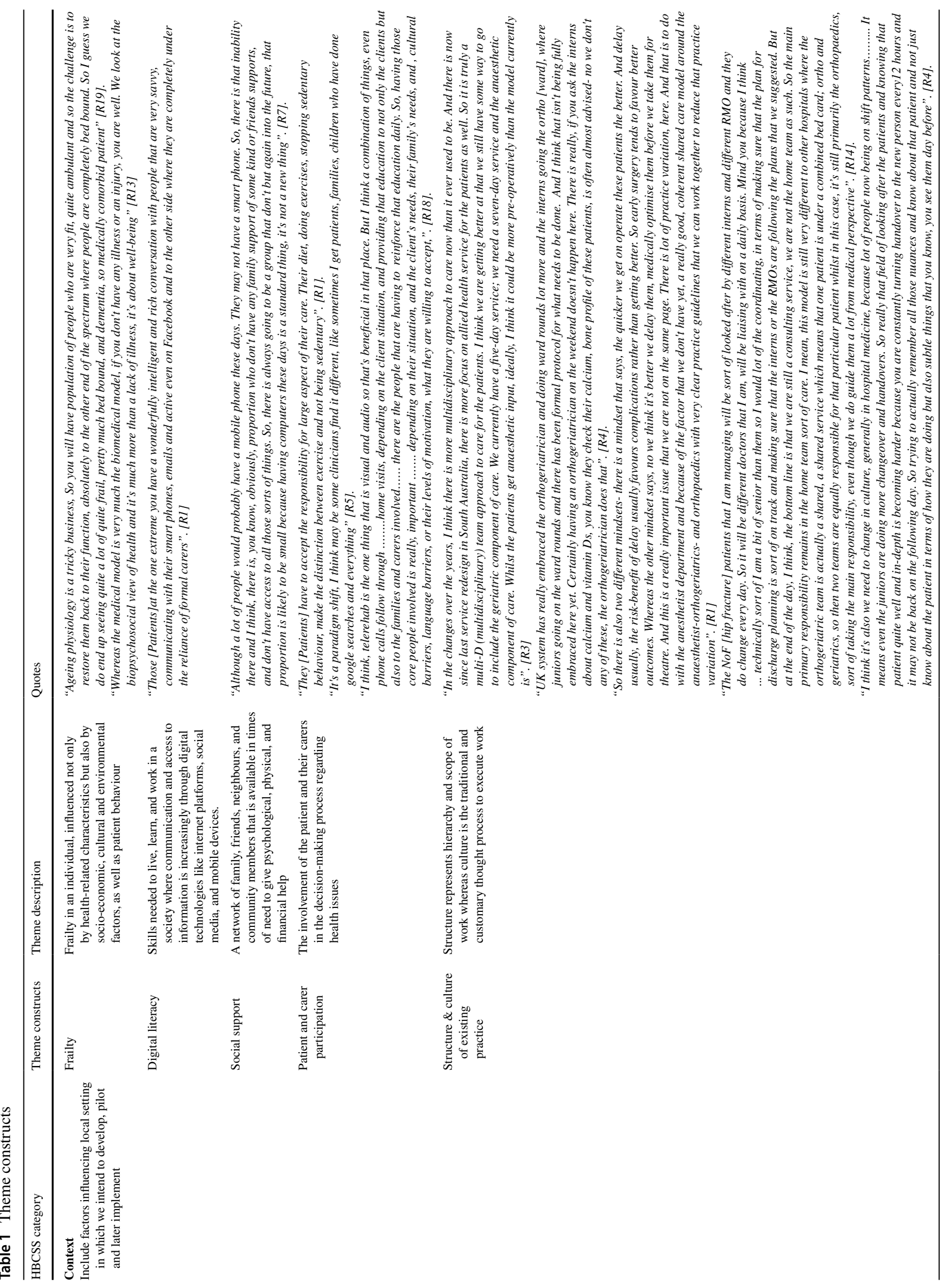




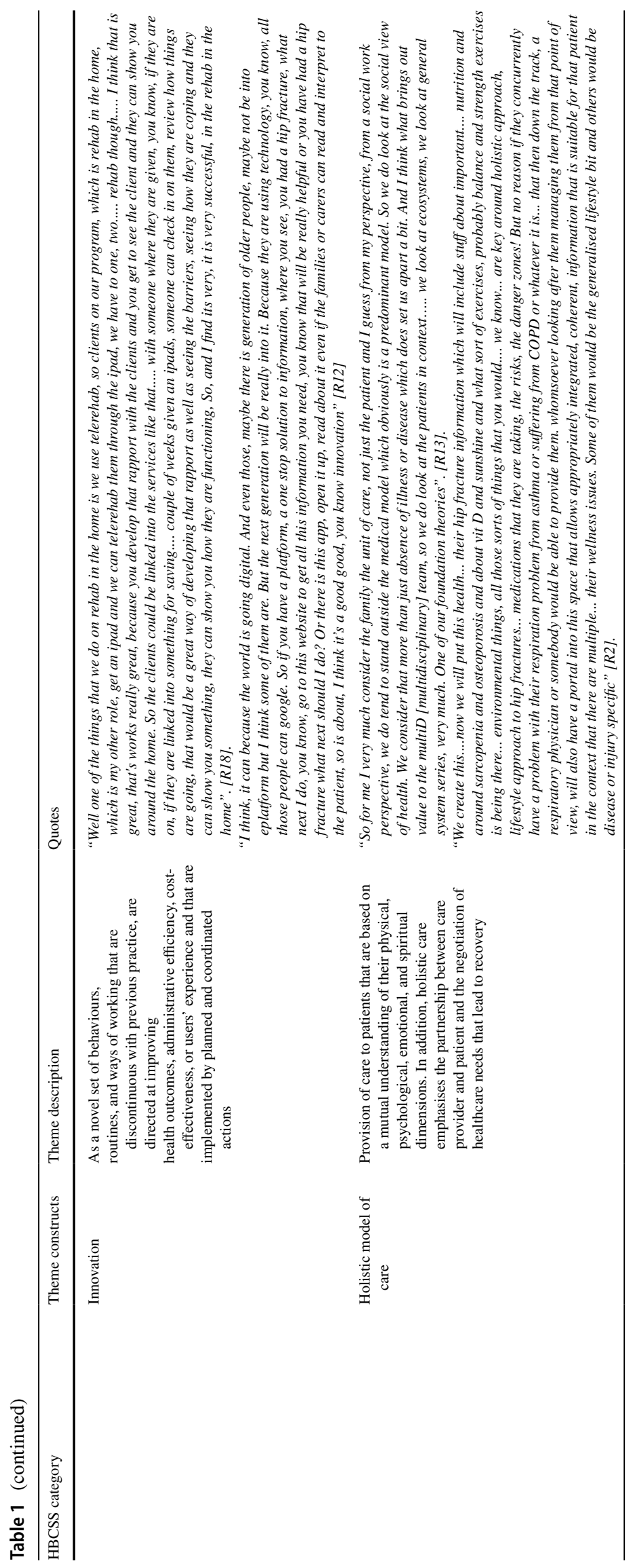




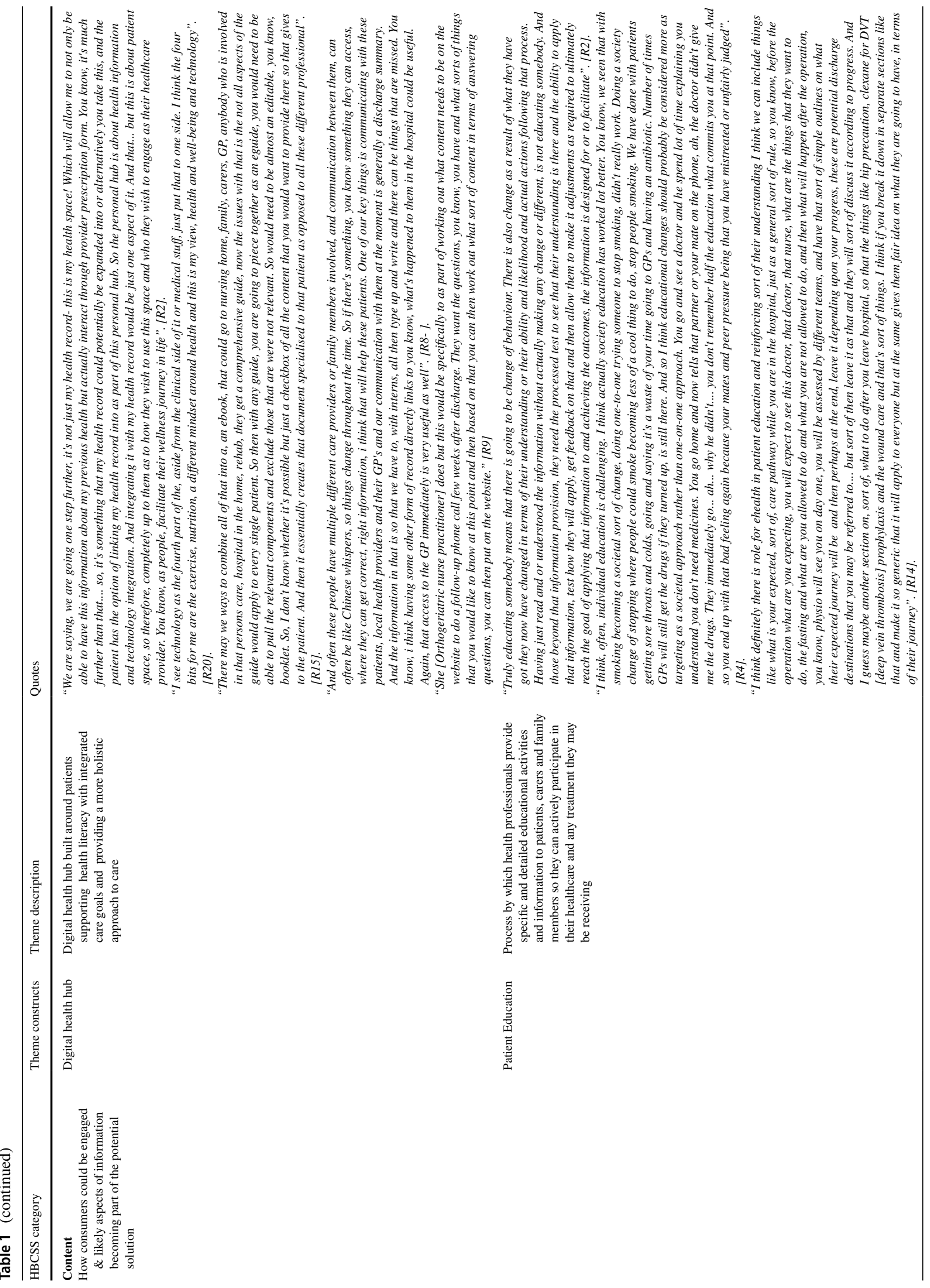




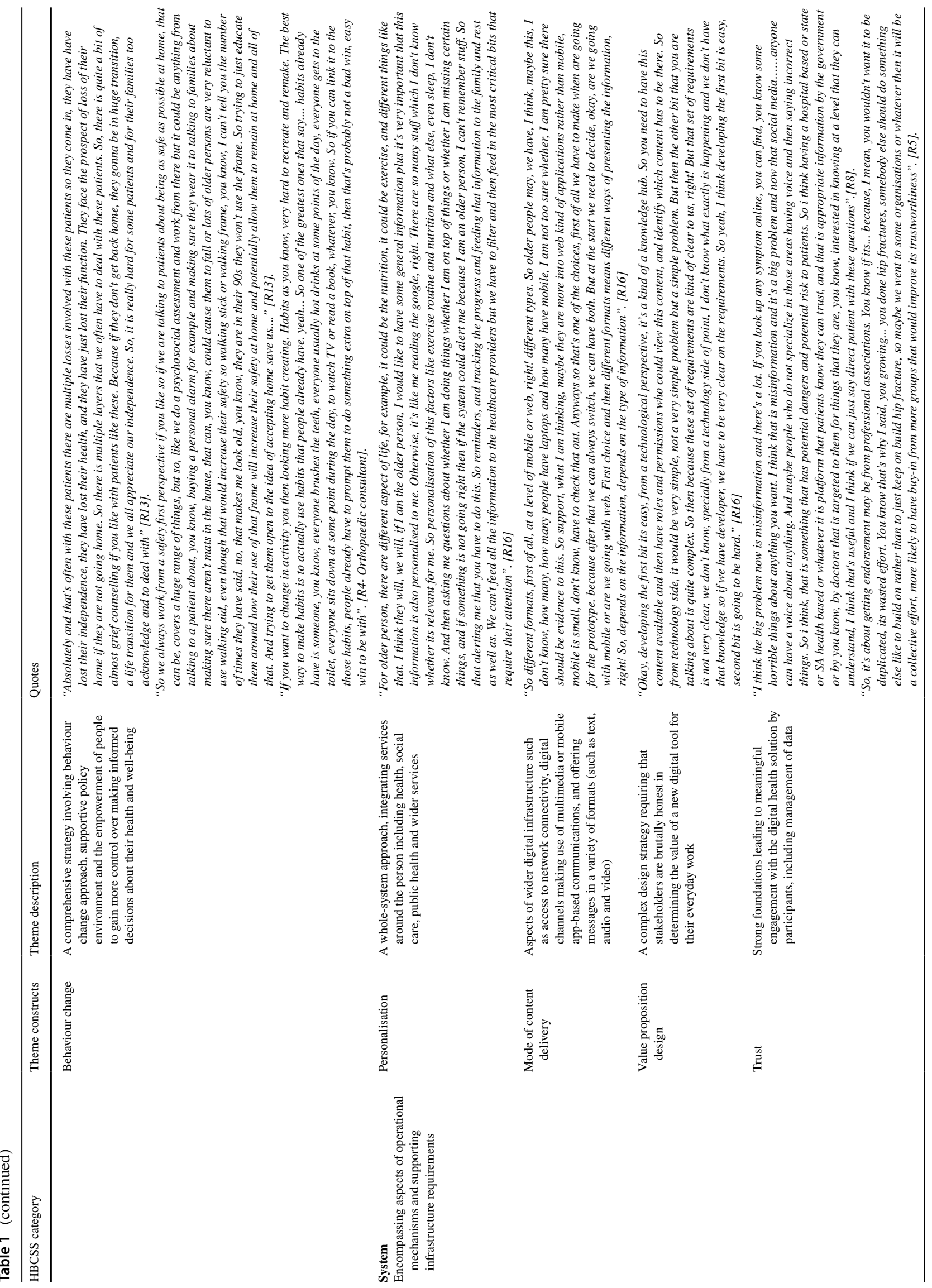




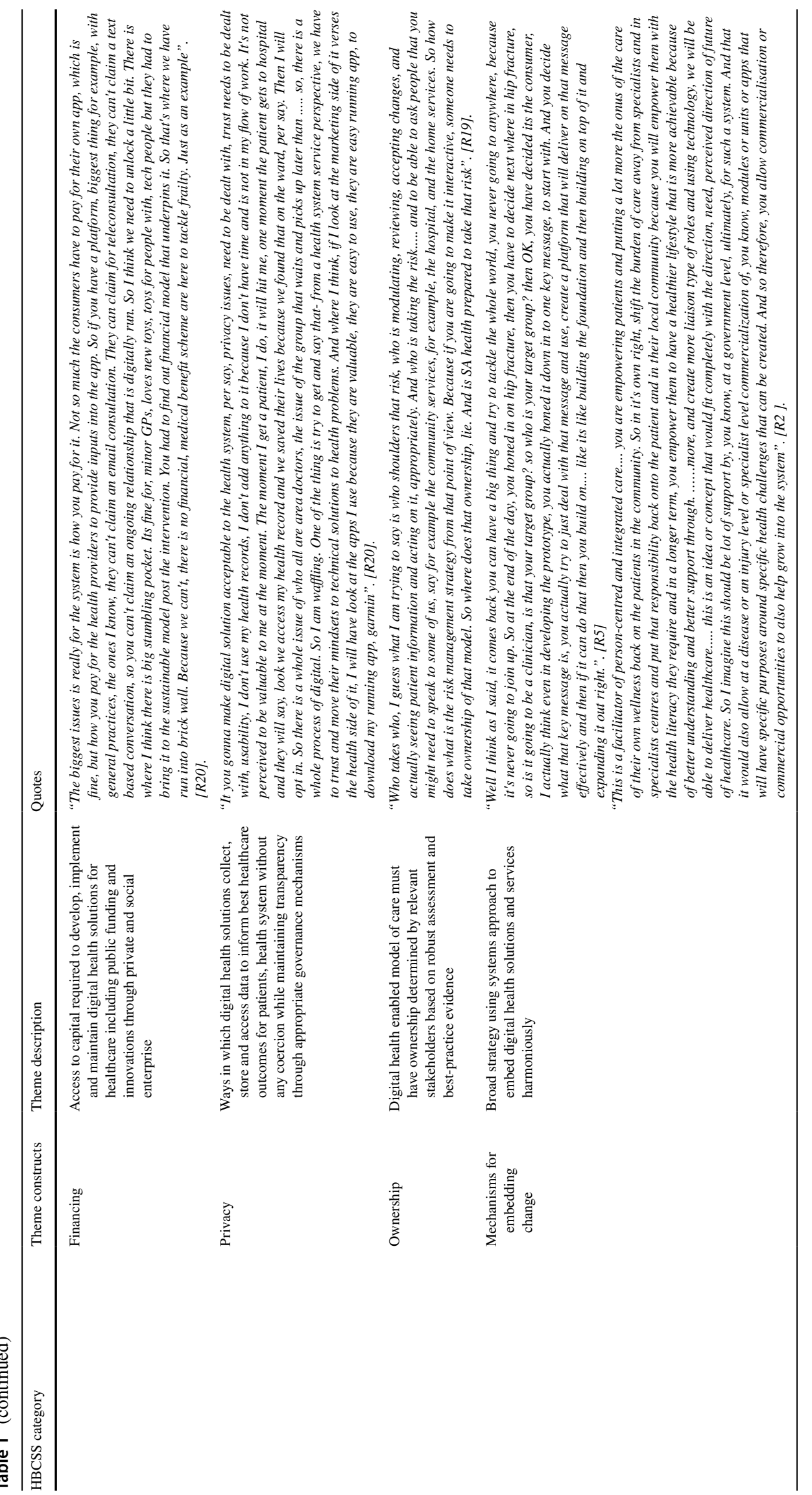


section are based on the further grouping of close thematic constructs under the categories of the HBCSS framework.

\section{Context}

\section{Frailty and digital literacy}

Study participants felt that hip fracture care is complex, reflects heterogeneity in terms of health and digital literacy, and is determined by the patient's intrinsic capacities and social circumstances. On one end of the spectrum, some patients are independent, whereas on the other end, they are frail, have poor nutritional status, and require permanent residential aged care support.

Participants agreed that digital literacy among patients is also variable; patients range from being technologically savvy to finding emerging technologies difficult to comprehend. Additionally, participants expressed the opinion that older people may not have access to smartphones, or the social support required to navigate such devices. However, participants felt that this scenario will change in the future, with older people more likely to have experience using technology.

\section{Patient/carer participation and social support}

Participants highlighted the empowerment of patients and their family members to take responsibility for patients' own health, especially in relation to exercise, nutrition, and the avoidance of sedentary behavior. By improving access to credible information and involving patients with their family members and designated carers, self-management and recovery could be optimized.

Participants felt that digital technologies would enable patients to better engage in conversations with their healthcare providers about their care processes. These solutions could range from telephone follow-up to interactive telerehabilitation guided by the expectations of patients and their family members and consider cultural and language preferences.

\section{Structure and culture of existing practice}

Study participants responsible for the coordination of multidisciplinary care mentioned that the model of care in the local setting is consistent with best practice evidence. However, there were significant gaps around improving the quality of healthcare and patient satisfaction. Some of the suggestions were process measures, primarily focused on the context outside of in-hospital care, the inclusion of minor fragility fractures, and the extension of multidisciplinary care from 5 to 7 days a week in the acute care setting. Participants also felt that there was a need to improve the coordination of care between different disciplines. An orthopedic consultant suggested that unlike the health system within the UK, the health system in Australia must embrace orthogeriatricians. Geriatric services must be available on weekends, as this may address missed opportunities, such as checking the bone profile of patients.

Another orthopedic consultant felt that there is a difference of opinion regarding delaying or not delaying surgery based on the trade-offs between ensuring better health outcomes versus preventing complications. Furthermore, it was highlighted that the role of the anesthetist must become part of a coherent shared care model aligning with best practice guidelines to reduce variations in the availability of anesthesia care across different practice settings.

The study participants also felt that there is a dilemma around how interns understand the care processes in comparison to the registrar within the orthogeriatric model of care due to their training orientation and experience over time. Although interns are an essential part of the orthogeriatric model of care, communication with patients often lacks the depth required to understand the nuances of coordinated care and patients' expectations due to frequent handovers as interns work in shifts. Participants suggested that patients expect uniform coordinated services across disciplines and are unaware of the subtle differences among healthcare practitioners. Participants also felt that besides hip fractures, there are other priority areas for the orthopedics department requiring more attention from the workforce. They opined that even with a dedicated orthopedic nurse practitioner looking after the multidisciplinary care coordination of hip fractures, staff from different disciplines may struggle to appreciate each other's roles and have conflicting priorities; thus, there is a need to improve mutual agreement over the care of these patients.

\section{Innovation and holistic model of care}

A study participant considered the implementation of a telerehab program at home to be innovative. Describing the experience, the participant emphasized rapport building with patients at the beginning of the process, tracking patients' progress over time, and connecting patients with other services. This type of approach was also supported by another participant who suggested that hip fracture needs to be considered beyond local tissue damage (medical model of care) and toward a more holistic biopsychosocial model of care. This involves taking into account the family as a whole unit of care. 


\section{Content}

\section{Digital health hub (DHH)}

When mentioning a patient electronic health record initiative recently rolled out across Australia (My Health Record), a study participant described the potential digital solution as a digital health hub. This hub could provide comprehensive care centered around patients through the provision of quality information and education and integrate different services as per the expectations of patients corresponding to their needs and circumstances. A general practitioner (GP) opined that a model of care supported by digital health would require embracing technological advancements, and such a change in mindset would help focus on overall health and wellbeing, including exercise and nutrition.

Study participants suggested different communication strategies for the hub where multiple care providers, including GP practices or family members, could be engaged through a trustworthy source. There could be an electronic booklet containing comprehensive information on various aspects of care, and relevant components could be pulled out and applied as per the needs of individual patients. An orthopedic registrar suggested that there are different care pathways where patient-centered communication is at the heart of coordinating services, which are often determined by the changing circumstances of the patient. Such a digital health hub could possibly overcome some of the gaps in existing mechanisms, such as with respect to the sharing of one-size-fits-all discharge summaries. A key care coordinator within the hip fracture context, such as an orthogeriatric nurse practitioner, could be engaged and interact with the content of the digital platform based on the follow-up requirements.

\section{Education and behavior change}

Patient education must adopt a biopsychosocial view of health rather than merely focusing on the hip fracture injury. Many of the participants argued that education does not equate to the provision of information. Educating patients is a process that involves taking into account their changed circumstances with respect to the injury and likely adjustments they will make on their care pathway. Education must include both patients and their family members, who already may be contemplating several changes and behavioral patterns during the transition process following a hip fracture injury.

A consultant suggested that health promotion and community education, which have worked in contexts such as controlling tobacco use or countering over prescription of antibiotics for the common cold, would yield better results than educating individuals. At times, the latter strategy may not work, as some patients return to the community and follow alternative advice from family members or friends. A clinical social worker had a different perspective around patient education and behavior change, suggesting that it is almost like counseling a patient who is on the brink of losing multiple things, ranging from functional independence to the house in which they spent their life prior to the fracture.

Digital health can be utilized to educate patients and their family members. This education can begin during the hospitalization period, informing them about the details of each care process within the care pathway. Essentially, this will help manage the expectations of patients and their family members. However, caution needs to be taken to avoid overwhelming patients with information that may not be appropriate to their stage of care.

\section{System}

\section{Personalization, mode of content delivery, and value proposition design}

With respect to the mode of content delivery, the participants believed that digital health solutions need to be personalized at the level of individual patients. The provision of information can be comprehensive, but information must align with the respective stage of care. A computer scientist suggested that developing a digital health knowledge hub may seem easy from the point of view of sourcing content and defining roles around access; however, operationalizing the hub would be complex. Therefore, there is a need to consider establishing effective mechanisms of communication and agreement over requirements and expectations between the software developer and relevant stakeholders responsible for designing and delivering the model of care.

A consultant in orthopedics suggested that information packages, if aligned with the regular habits of the individual, can be more effective than trying to create a new habit or routine. A range of different formats, such as texts (written information in the form of existing handouts or electronic reading), podcasts, and multimedia (videos or interactive solutions to facilitate the active involvement of an individual who can interact and exercise choices), need to be developed for the optimal engagement of patients and their carers on several aspects of care while maintaining general health. Another participant suggested the emerging role of avatarslife-like characters that can digitally interact with patients or become part of virtual reality solutions. The digital health platform could also be a resource center for healthcare practitioners, particularly those at the frontline, including junior doctors. 


\section{Trust, financing, privacy, and ownership}

There were some areas highlighted by the participants requiring further debate and discussion. These included financing the digital health hub, essentially from the perspective of different healthcare providers. Experiences shared by a GP suggested that intricacies within telemedicine require careful consideration. For instance, compensation is provided for teleconsultation but not for any ongoing digital interactions such as emails or text-based conversations. Furthermore, this participant suggested that when piloting the digital health hub, GPs would be enthusiastic and support the initial implementation phase; however, after the initial pilot, a roadmap should indicate not only how this will be scaled up but also how it will be financed and incentivized.

Participants agreed that internet access is likely to continue to increase in the future, and people may become confused at times due to information overload and misinformation. Thus, there is a need for an authentic digital health solution that could address these issues and guide people in the right direction to access accurate information. A geriatrician suggested that endorsement by professional associations would likely improve information trustworthiness and possibly reduce duplicate efforts by mutual sharing, enabling people to build upon each other's learning. There is also a need to shift the mindset of healthcare providers toward technology-focused solutions wherever possible.

Similarly, another geriatric consultant believed that ownership issues should be addressed once a digital health solution is implemented within the healthcare system. Risks associated with accessing patient information and acting upon it, modulating, reviewing patient education resources, and tracking progress would require careful safeguards.

\section{Mechanisms for embedding change}

The study participants believed an appropriate mechanism needs to be in place to allow different stakeholders to interact with the digital platform uniformly and seamlessly according to their role in the hip fracture care pathway. Their engagement also involves assessing patients' health and health literacy and providing them support wherever possible. In participants' opinion, deploying this type of innovative digital health platform would involve intricate processes that would enable positive changes to hip fracture care and improve patient outcomes. It would include communicating with a software developer in a language that can be understood and that is based on mutually agreed-upon priorities. A solution-focused approach, instead of one-way communication or direction, should guide collaboration. It was also suggested by participants that the engagement of experts in education and computer science must be consistent throughout the development, piloting and wider roll-out of a digital health model of care. Furthermore, the participants commented that the digital health content must be easy to understand. It must be assumed that patients and their carers engaging with this platform have a primary education level, and the platform must align with their dayto-day activities.

Another participant suggested that although the intent is to develop a comprehensive digital health solution, it is important to consider the development process in stages, similar to the way a house is built. Study participants reinforced the notion that it is critically important to keep patients at the center of care by empowering them through healthcare practitioners. The platform could also help upskill healthcare practitioners through a mutual learning process. With these embedding mechanisms in place, new liaising roles would be created using technology to deliver new models of care and behavior change.

\section{Discussion}

This study involved the perspectives of various stakeholders from multiple disciplines, including clinical, allied health, computer science, education, research, and policy. It contributes to an ongoing program of research focused on a personalized digital health hub (DHH)-enabled model of care facilitating recovery from fragility fractures and potentially other health conditions $[1,4,5]$. We followed a knowledge translation approach to engage people who could influence micro (clinical practice), meso (health setting), and macro levels (thought leaders influencing policy and research agenda). Some of these stakeholders could remain key players during the next stage of implementation, facilitating the integration of the digital health hub and advocating for a person-centered integrated model of healthcare delivery. The findings from this study were classified into 18 thematic constructs embedded within three categories, context, content, and system, which correspond to a theoretical human-computer persuasive systems framework, HBCSS [4, 12]. These thematic constructs covered a range of patient factors, including the role of family members, social support, and the characteristics of existing healthcare delivery, as well as the enabling of computing functionalities and requirements to operate within a multidisciplinary environment.

Stakeholders within our study agreed that new models of care using digital health could strengthen the existing hip fracture care pathway. However, collectively, all of the different healthcare providers associated with the care pathway must take into account patients' needs and outcome expectations, moving beyond the medical model focused on the repair of local tissue damage. Overwhelmingly, 
contemporary evidence suggests that a significant number of hip fracture patients, once admitted to acute care hospitals, do not return to their pre-fracture residence and are unable to attain optimal health status $[2,20]$. Thus, an MoC must go beyond clinical practice guidelines and incorporate practical delivery issues regarding for whom, when, where, and how care is best delivered and re-evaluated [21]. This can become complex due to a multidisciplinary workforce, links with secondary and tertiary care services, the biopsychosocial needs of the patients, and frequently changing organizational structures. However, our study findings take a step further, highlighting that mere change within the organizational structure is not sufficient, and a new mindset embracing technological advancement is crucial for shifting an organizational culture toward envisioning a holistic model of healthcare delivery. There are huge gaps; for instance, primary care offers a generalist approach, and while older adults with multimorbidities represent a large proportion of GP consultations, the required access to other community services is patchy $[6,21]$. It is often left to the patients and their carers to coordinate and navigate the range of services, which could be influenced by their social circumstances and priorities [21]. Thus, patient education and the provision of accurate, timely, and adequate information could play a vital role in improving engagement and participation in the recovery or rehabilitation process within a $\mathrm{MoC}$ [8]. The DHH-enabled MoC could potentially be a powerful tool empowering patients to take greater control of their health goal(s). Moreover, the processes within a DHH can link patients to a more extensive network of specialists, general practitioners, and community carers $[4,5]$.

According to the World Health Organization (WHO) Integrated Care for Older People (ICOPE) guidelines, when working toward solutions for older people in the community, we need to acknowledge that older people's health is on a wide spectrum, ranging from perfectly healthy to moribund status based on their "decline in the intrinsic capacities" [15, 22]. Our findings from this study also suggest that future healthcare must focus on an evolving aging physiology, yet the current understanding within the practice environment may be inadequate to fully incorporate this view. The care processes become complex due to different interacting components defined by each patient's unique needs and sets of expectations as well as the health workforce capacity. Our study findings suggest that the coordination and integration of multiple disciplines would require innovation and the effective engagement of patients along with their family members as a whole unit of care. A similar view is supported by some of the contemporary guidelines and recommendations around innovation diffusion and health workforce innovations [23-26].

Musculoskeletal conditions, including fragility fractures, are among the key contributors to the global burden of disease [27] and are predicted to increase, in terms of both their impact and prevalence, due to an aging population and the increased prevalence of noncommunicable diseases $[27,28]$. Emerging evidence suggests that digital health interventions improve care outcomes for fragility fractures, osteoarthritis, and chronic low back and neck pain [29-35]. Nevertheless, critical questions need to be raised around what constitutes a digital health "intervention package," and its effectiveness must be tested pragmatically according to the local implementation context [1]. The debate around patients' engagement in their own care has predominantly remained within the confines of narrow pilot research projects or at the level of a philosophical argument without full realization of its practical effectiveness [36]. This may be attributed to a misguided perception of engagement strategies such as cocreation and/or coproduction, which essentially represent "values" and not necessarily intervention strategies [36]. Therefore, while we consider these value-based strategies an important way to empower patients, their family members, and healthcare providers together, understanding other aspects of complexity within an MoC would drive successful implementation [36-38].

Access to technology is a multifaceted factor, as there is likely to be a digital divide due to varied levels of e-literacy or an inability to trust and navigate the plethora of information available online [9]. Existing digital health solutions target healthcare providers and consumers differently. Electronic health records mainly allow communication, data management, and clinical decision support among healthcare providers; these records are not primarily meant to engage patients [39]. The Australian My Health Record (MyHR) is a national, personally controlled electronic health record that can track the journey of patients within the healthcare system through a shareable summary of an individual's health information [39]. MyHR envisages that different healthcare practitioners will eventually move away from the traditional "push" method of accessing patient medical information and towards a "pull" method of information sharing [40]. The latter approach allows healthcare practitioners to access relevant information by pulling information from individual health records rather than pushing a request to other care providers for similar information [40, 41]. A similar change in consumer behavior can be seen in relation to accessing current affairs information. Consumers are rapidly shifting from using conventional media platforms that push current affairs information through television sets to digital platforms such as YouTube or exclusive interactive platforms. The latter multimedia platforms have enabled consumers to pull information according to their choice, thus forcing the content creator to ensure quality, authenticity, and a safe interactive environment [42]. Nevertheless, one must also learn from the recent rollout of MyHR in Australia that 
despite a $90 \%$ subscription rate by patients, less than a quarter of healthcare providers were using the system. The top priorities for this system have been clinical integration and the improvement of patient experiences; however, there could be a significant gap in terms of educating practitioners to use digital systems and facilitate patient engagement. Acknowledging these gaps and working around effective solutions could incrementally improve patient outcomes and service delivery [7]. The findings from our study highlight the complexity of the financing potential digital health hubs, privacy, ownership, and the establishment of trust along with the need to create new business models. Different business models utilizing digital technologies are now emerging in the commerce, entertainment, and media sectors and are demonstrating success with financing, trust, and privacy. This gives us an opportunity to discuss how these business models can be adopted and best translated into health settings [43, 44].

This study involved an in-depth interview methodology with a wide range of stakeholders to map out potential factors that could influence the design and implementation of a DHH-enabled MoC for fragility hip fractures within a local healthcare setting. We did not recruit participants from other local health networks within the region, which could limit the generalizability of our findings, particularly in relation to application across South Australia or Australia. There is a digital divide posed by challenges due to geography, internet availability and uninterrupted internet access, people living with disabilities, and factors related to sociopolitical and economic disparities. However, some of the participants in this study had experience in diverse settings, including other developed countries and emerging economies. We believe this diversity enhanced the richness of the data and provided wider applicability of findings in terms of opening lines for multiple inquiries and future research areas. Our study also attempted to integrate early on the perspectives of frontline healthcare delivery staff and the research context, aligning with the implementation strategies for the next stage of the research program $[1,4,9]$.

\section{Conclusion}

Our study, which was conducted before the COVID-19 pandemic, highlighted that the organizational structure and existing practice culture across the health system may resist change when introducing digital healthcare. However, as we are living through the current pandemic, the use of digital technologies has not only accelerated the willingness of healthcare practitioners and organizations to adopt telehealth options but also improved the engagement of consumers [7]. Compared to the last decade, there has been a rapid increase in the availability of digital health solutions by a variety of vendors, including health agencies, fitness advocates, and software companies. These applications are primarily designed to support consumers in the domain of general lifestyle and wellness, while some have been developed to support selfmanagement of chronic disease conditions such as diabetes $[5,18,45]$. At the time when this study was conducted, we only had a theoretical concept of a digital health solution to be co-designed with relevant stakeholders. However, with critical insights from this study, including the characterization of DHH and findings from other research components $[1,4,5,7$, 9], we have been able to advance our concept to the stage of a prototype to be tested in a real-world practice setting in the next stage of our research program. The DHH-enabled MoC will be structured to provide information related to not only hip fractures but also general health and wellbeing, including nutrition, exercise, sleep, and mental health, combined with past medical history [5]. We recommend wider consultations across a range of practice environments or specialty groups so that tailored innovative services can be negotiated, developed, and tested for implementation.

Supplementary Information The online version contains supplementary material available at https://doi.org/10.1007/s11657-021-01031-3.

Acknowledgements The authors would like to acknowledge all of the study participants from CALHN and other stakeholders for their time, effort, and valuable perspectives shared through in-depth interviews, without which this study could not have been possible.

Author contribution LY, MJC, and TKG contributed to the conceptualization of the study and the design of the interview guide. LY conducted the in-depth interviews, the verbatim transcription, and the analysis, and engaged in routine discussion with TKG and MJC. LY produced the first draft. TKG, MJC, RV, AT, and JY reviewed the draft and provided critical inputs in relation to the thematic constructs and overall flow of the manuscript. Subsequent revisions of the draft followed. This final version of the manuscript incorporates comments and edits from the authors and is approved by all authors.

Funding This study is financially supported by NHMRC Centre for Research Excellence (CRE) in Frailty and Healthy Ageing at the Adelaide Medical School, the University of Adelaide (NHMRC Grant ID- 1102208). LY is supported by the Commonwealth Government of Australia funded Research Training Program Scholarship and Top-up scholarships through NHMRC CRE-Frailty \& Healthy Ageing. There was no support from any other external sources.

\section{Declarations}

Ethics Ethics approval for the study was provided by the Human Research Ethics Committee of the Central Adelaide Local Health Network (Ref: R20181005, HREC/18/CALHN/687). Governance review and authorization were granted by the Royal Adelaide Hospital (SSA/19/CALHN/59, Ref: 11183) and The Queen Elizabeth Hospital (SSA/19/CALHN/57, Ref: 11184) and further approved by the Human Research Ethics Secretariat and Legal and Risk Office of the University of Adelaide (Ref: 3338).

Conflicts of interest None. 


\section{References}

1. Yadav L, Haldar A, Jasper U, Taylor A, Visvanathan R, Chehade $\mathrm{M}$ et al (2019) Utilising digital health technology to support patient-healthcare provider communication in fragility fracture recovery: systematic review and meta-analysis. Int J Environ Res Public Health 16(20):4047. https://doi.org/10.3390/ijerph1620 4047

2. Jorissen RN, Lang C, Visvanathan R, Crotty M, Inacio MC (2020) The effect of frailty on outcomes of surgically treated hip fractures in older people. Bone 136:115327. https://doi.org/10.1016/j.bone. 2020.115327

3. Williamson S, Landeiro F, McConnell T, Fulford-Smith L, Javaid MK, Judge A, Leal J (2017) Costs of fragility hip fractures globally: a systematic review and meta-regression analysis. Osteoporos Int 28(10):2791-2800. https://doi.org/10.1007/ s00198-017-4153-6

4. Yadav L, Gill TK, Taylor A et al (2019) Cocreation of a digital patient health hub to enhance education and person-centred integrated care post hip fracture: a mixed-methods study protocol. BMJ Open: e033128. https://doi.org/10.1136/bmjop en-2019-033128

5. Chehade MJ, Yadav L, Jayatilaka A, Gill TK, Palmer E (2020) Personal digital health hubs for multiple conditions. Bull World Health Organ 98(8):569-575. https://doi.org/10.2471/BLT.19. 249136

6. Tomasdottir MO (2017) Multimorbidity in the Norwegian HUNT population: an epidemiological study with reference to the concept allostatic load [Doctoral thesis]. Trondheim, Norwegian University of Science and Technology (NTNU) Grafisk senter. https:// opinvisindi.is/bitstream/handle/20.500.11815/473/Thesis-final MOT.pdf?sequence=1\&isAllowed=y. Accessed 20 Sept 2020

7. Yadav L, Gill TK, Taylor A, De Young J, Chehade MJ (2021) Identifying opportunities, and motivation to enhance capabilities, influencing the development of a personalized digital health hub model of care for hip fractures: mixed methods exploratory study. J Med Internet Res 23(10):e26886. https:// doi.org/10.2196/26886

8. Chehade MJ, Gill TK, Kopansky-Giles D, Schuwirth L, Karnon J, McLiesh P et al (2016) Building multidisciplinary health workforce capacity to support the implementation of integrated, people-centred Models of Care for musculoskeletal health. Best Pract Res Clin Rheumatol 30(3):559-84. https://doi.org/10.1016/j. berh.2016.09.005

9. Chehade MJ, Yadav L, Kopansky-Giles D, Merolli M, Palmer E, Jayatilaka A, Slater H (2020) Innovations to improve access to musculoskeletal care. Best Pract Res Clin Rheumatol 34(5):101559. https://doi.org/10.1016/j.berh. 2020.101559

10. Shirooka H, Nishiguchi S, Fukutani N et al (2017) Association between comprehensive health literacy and frailty level in community-dwelling older adults: a cross-sectional study in Japan. Geriatr Gerontol Int 17:804-809

11. Richtering SS, Morris R, Soh S-E et al (2017) Examination of an eHealth literacy scale and a health literacy scale in a population with moderate to high cardiovascular risk: Rasch analyses. PLoS One 12:e0175372

12. Kelders SM, Oinas-Kukkonen H, Oörni A et al (2016) Health behaviour change support systems as a research discipline; a viewpoint. Int J Med Inform 96:3-10

13. Harvey G, Kitson A (2015) PARIHS revisited: from heuristic to integrated framework for the successful implementation of knowledge into practice. Implement Sci 11:33. https://doi.org/10.1186/ s13012-016-0398-2
14. National Clinical Guideline Centre (2017) The management of hip fractures in adults. National clinical guideline centre (2017 addendum). Available: www. ncgc. ac. Uk

15. World Health Organization (2017) Integrated care for older people: guidelines on community-level interventions to manage declines in intrinsic capacity. World Health Organization, Geneva (Licence: CC BY-NC-SA 3.0 IGO)

16. Harwell MR (2011) Research design in qualitative/quantitative/ mixed methods. In: Conrad CF, Serlin RC (eds) The SAGE Handbook for research in education: pursuing ideas as the keystone of exemplary inquiry. SAGE Publications, Inc, Thousand Oaks, pp $147-82$

17. Tong A, Sainsbury P, Craig J (2007) Consolidated criteria for reporting qualitative research (COREQ): a 32-item checklist for interviews and focus groups. Int J Qual Health Care 19(6):349-357

18. Fereday J, Muir-Cochrane E (2006) Demonstrating rigor using thematic analysis: a hybrid approach of inductive and deductive coding and theme development. Int J Qual Methods 5(5):80-92. https://doi.org/10.1177/160940690600500107

19. QSR International Pty Ltd. (2018) NVivo (Version 12). https:// www.qsrinternational.com/nvivo-qualitative-data-analysis-softw are/home. Accessed 20 Sept 2020

20. Mitchell R, Draper B, Harvey L, Wadolowski M, Brodaty H, Close J (2019) Comparison of hospitalised trends, treatment cost and health outcomes of fall-related hip fracture for people aged $\geq 65$ years living in residential aged care and the community. Osteoporos Int 30(2):311-321. https://doi.org/10.1007/ s00198-018-4800-6

21. Dziedzic KS, French S, Davis AM, Geelhoed E, Porcheret M (2016) Implementation of musculoskeletal models of care in primary care settings: theory, practice, evaluation and outcomes for musculoskeletal health in high-income economies. Best Pract Res Clin Rheumatol 30(3):375-397. https://doi.org/10.1016/j.berh. 2016.08.004

22. World Health Organization (WHO) (2015) World Report on Ageing and Health. Switzerland, Geneva

23. Greenhalgh T, Robert G, Macfarlane F, Bate P, Kyriakidou O (2004) Diffusion of innovations in service organizations: systematic review and recommendations. Milbank Q 82(4):581-629. https://doi.org/10.1111/j.0887-378X.2004.00325.x

24 Greenhalgh T, Robert Greenhalgh T, Robert G, Macfarlane F et al (2004) Diffusion of innovations in service organizations: systematic review and recommendations. Milbank Q 82:581e629

25. World Health Organization (2016) Global strategy on human resources for health: workforce 2030. WHO. https://apps. who. int/iris/bitstream/handle/10665/250368/9789241511131-eng.pdf? sequence.1. Accessed 20 Sept 2020

26. Britnell M (2019) Human: solving the global workforce crisis in healthcare. Oxford Scholar. https://doi.org/10.1093/oso/97801 98836520.001 .0001

27. James SL, Abate D, Abate KH et al (2018) GBD 2017 disease and injury incidence and prevalence collaborators. Global, regional, and national incidence, prevalence, and years lived with disability for 354 diseases and injuries for 195 countries and territories, 1990-2017: a systematic analysis for the Global Burden of Disease Study 2017. Lancet 392(10159): 1789e858

28. World Health Organization (2019) Musculoskeletal conditions. 26th November. https://www.who.int/news-room/factsheets/detail/ musculoskeletal-conditions. Accessed 20 Sept 2020

29 Slater H, Dear BF, Merolli MA et al (2016) Use of eHealth technologies to enable the implementation of musculoskeletal Models of Care: evidence and practice. Best Pract Res Clin Rheumatol 30(3):483e502. https://doi.org/10.1016/j.berh.2016.08.006

30. Slater H, Campbell JM, Stinson JN et al (2017) End user and implementer experiences of mHealth technologies for 
noncommunicable chronic disease management in young adults: systematic review. J Med Internet Res 19(12):e406. https://doi. org/10.2196/jmir.8888

31 Grasaas E, Fegran L, Helseth S et al (2019) iCanCope with pain: cultural adaptation and usability testing of a self-management app for adolescents with persistent pain in Norway. JMIR Res Protoc 8(6):e12940. https://doi.org/10.2196/12940

32. Slater H, Jordan JE, Houlding E et al (2019) painHEALTH evaluation report executive summary. Department of Health, Health Networks Government of Western Australia; https://ww2.health.wa. gov.au/ /media/Files/Corporate/general\%20documents/Health\% 20Networks/Musculoskeletal/painHEALTH-Evaluation-ReportExecutive-Summary.pdf. Accessed 20 Sept 2020

33. Stinson JN, Lalloo C, Harris L et al (2014) iCanCope with Pain: user-centred design of a web- and mobile-based self-management program for youth with chronic pain based on identified health care needs. Pain Res Manag 19(5):257e65

34. Cottrell MA, Galea OA, O'Leary SP et al (2017) Real-time telerehabilitation for the treatment of musculoskeletal conditions is effective and comparable to standard practice: a systematic review and meta-analysis. Clin Rehabil 31(5):625e38. https://doi.org/10. $1177 / 0269215516645148$

35 Lawford BJ, Hinman RS, Nelligan RK et al (2019) "I could do it in my own time and when I really needed it": perceptions of online pain coping skills training for people with knee osteoarthritis. Arthritis Care Res. https://doi.org/10.1002/acr.24093

36. Voorberg WH, Bekkers VJJM, Tummers LG (2015) A systematic review of co-creation and co-production: embarking on the social innovation journey. Public Manag Rev 17(9):1333-1357. https:// doi.org/10.1080/14719037.2014.930505

37. O'Cathain CL, Sworn K et al (2019) Taxonomy of approaches to developing interventions to improve health: a systematic methods overview. Pilot Feasibility Stud 5:41. https://doi.org/10.1186/ s40814-019-0425-6

38. Turner KM, Rousseau N, Croot L, Duncan E, Yardley L, O'Cathain A, Hoddinott P (2019) Understanding successful development of complex health and healthcare interventions and its drivers from the perspective of developers and wider stakeholders: an international qualitative interview study. BMJ Open 9:e028756. https://doi.org/10.1136/bmjopen-2018-028756

39. Walsh L, Hemsley B, Allan M et al (2019) Assessing the information quality and usability of My Health Record within a health literacy framework: what's changed since 2016? Health Inf Manag J. https://doi.org/10.1177/1833358319864734

40. Kariotis T, Prictor M, Chang S, Gray K (2019) Evaluating the contextual integrity of Australia's My Health Record. Stud Health Technol Inform 265:213-218. https://doi.org/10.3233/SHTI1 90166

41. Pang PC, Chang S (2019) The Twitter Adventure of \#MyHealthRecord: an analysis of different user groups during the opt-out period. Stud Health Technol Inform 266:142-148. https://doi.org/ 10.3233/SHTI190786

42. Wilding D, Fray P, Molitorisz S, McKewon E (2018) The impact of digital platforms on news and journalistic content. University of Technology Sydney, NSW

43. Evens T, Raats T, von Rimscha MB (2017) Business model innovation in news media organisations - 2018 special issue of the European Media Management Association (emma). J Media Bus Stud 14(3):167-172. https://doi.org/10.1080/16522354.2018. 1445164

44. Horst SO, Järventie-Thesleff R, Baumann S (2019) The practice of shared inquiry: how actors manage for strategy emergence. J Media Bus Stud 16(3):202-229. https://doi.org/10.1080/16522 354.2019 .1641672

45 McKay FH, Wright A, Shill J, Stephens H, Uccellini M (2019) Using health and well-being apps for behavior change: a systematic search and rating of apps. JMIR Mhealth Uhealth 7(7):e11926. https://doi.org/10.2196/11926

Publisher's note Springer Nature remains neutral with regard to jurisdictional claims in published maps and institutional affiliations.

\section{Authors and Affiliations}

\section{Lalit Yadav ${ }^{1,2,3} \cdot$ Tiffany K. Gill ${ }^{3} \cdot$ Anita Taylor ${ }^{2} \cdot$ Jennifer deYoung ${ }^{2} \cdot$ Renuka Visvanathan $^{1,3,4} \cdot$ Mellick J. Chehade $^{1,2,3}$}

Tiffany K. Gill

Tiffany.gill@adelaide.edu.au

Anita Taylor

Anita.taylor@sa.gov.au

Jennifer deYoung

Jen.deYoung@sa.gov.au

Renuka Visvanathan

Renuka.visvanathan@adelaide.edu.au

Mellick J. Chehade

Mellick.chehade@adelaide.edu.au
1 National Health and Medical Research Council Centre for Research Excellence in Frailty and Healthy Ageing, Adelaide Medical School, the University of Adelaide, Adelaide, Australia

2 Discipline of Orthopaedics \& Trauma, Royal Adelaide Hospital, Level 5G, 581, North Terrace, Adelaide, SA 5000, Australia

3 Adelaide Medical School, Faculty of Health and Medical Sciences, the University of Adelaide, Adelaide, Australia

4 Aged and Extended Care, The Queen Elizabeth Hospital, Central Adelaide Local Health Network, Adelaide, Australia 